\title{
"NECESSARY AND SUFFICIENT CONDITIONS FOR THE ROOTS OF A CUBIC POLYNOMIAL AND BIFURCATIONS OF CODIMENSION-1, -2, -3 FOR 3D MAPS"
}

- Laura Gardini (Department of, Economics, Society and Politics, University of Urbino, Italy)

- Noemi Schmitt (Department of Economics, University of Bamberg, Bamberg, Germany)

- Iryna Sushko (Institute of Mathematics, NASU, and Kyiv School of Economics, Kyiv, Ukraine)

- Fabio Tramontana (Department DiMSEFA, Università Cattolica del Sacro Cuore, Milano, Italy)

- Frank Westerhoff (Department of Economics, University of Bamberg, Bamberg, Germany) 


\title{
Necessary and sufficient conditions for the roots of a cubic polynomial and bifurcations of codimension-1, $-2,-3$ for 3D maps
}

\author{
LAURA GARDINI ${ }^{1}$ \\ Department DESP, University of Urbino, Urbino, Italy, laura.gardini@uniurb.it \\ Noemi Schmitt \\ Department of Economics, University of Bamberg, Bamberg, Germany, noemi.schmitt@uni-bamberg.de \\ IRYNA SushKo \\ Institute of Mathematics, NASU, and Kyiv School of Economics, Kyiv,Ukraine, sushko@imath.kiev.ua \\ Fabio Tramontana \\ Department DiMSEFA, Università Cattolica del Sacro Cuore, Milano, Italy, fabio.tramontana@unicatt.it \\ Frank WesterhofF \\ Department of Economics, University of Bamberg, Bamberg, Germany, frank.westerhoff@uni-bamberg.de
}

\begin{abstract}
We reconsider the well-known Schur/Samuelson conditions, which guarantee the roots of a third-degree polynomial to be inside the unit circle. These conditions are important in the stability analysis of equilibria and cycles of three-dimensional systems in discrete time. We derive a simplified set of conditions that determine the boundary of the stability region and prove which kind of bifurcation occurs when the boundary is crossed at any of its points. These points correspond to the existence of one, two or three eigenvalues equal to 1 in modulus, real or complex conjugate, and all the remaining eigenvalues are also explicitly given. The results are applied to a system representing a housing market model that gives rise to a Neimark-Sacker bifurcation, a flip bifurcation or a pitchfork bifurcation.
\end{abstract}

Keywords: Roots of a cubic polynomial, Necessary and sufficient conditions, Nonlinear Economic Dynamics, Stability conditions, Three-dimensional maps, Codimension-1,-2,-3 bifurcations.

JEL classification: C02, C61, C62, C65

\section{Introduction}

Low-dimensional discrete dynamical systems are studied extensively in many economic fields, ranging from microeconomics to macroeconomics and finance. In particular, the stability of equilibria in those systems is often investigated with the goal to control or maintain some desired behavior. For seminal contributions and illuminating applications, see, for instance, Anufriev and Bottazzi (2010), Asano and Yokoo (2019), Brock et al. (2005), Brock and Hommes (1997), Invernizzi and Medio (1991), Matsuyama (1999) and Matsuyama et al. (2016). The edited volumes by Tesfatsion and Judd (2006), Puu and Sushko (2006), Bischi et al. (2009), Hens and Schenk-Hoppé (2009), Rosser (2009) and Hommes and LeBaron (2018) contain further examples, while we refer the reader to Day (1994), Shone (2002), Puu (2004), Galor (2007), Hommes (2013), Miao (2014) and Sorger (2015) for general introductions to this line of research. Since the stability and bifurcation analysis of one- and two-dimensional systems is well known, the bulk of the aforementioned work is concentrated in that area. Unfortunately, a comparable formal treatment of three-dimensional systems is less trivial. For this reason, only few examples exist, including Agliari et al. (2000), Lines (2005), Bischi and

\footnotetext{
${ }^{1}$ Corresponding author.
} 
Tramontana (2010), Tramontana et al. (2010), Dieci and Westerhoff (2010), Matsumoto and Szidarovszky (2014), Naimzada and Tramontana (2015) and Cavalli et al. (2017). This as a real pity because threedimensional systems facilitate a more developed modeling of economic reality than is the case with one- or two-dimensional systems, and - in our view - thus deserve more attention. In this paper, we pave the way for more work in this direction by providing helpful tools to study such systems.

Many scholars know the celebrated work by Farebrother (1973) and use the four conditions mentioned therein. These conditions are equivalent to the necessary and sufficient conditions by Schur and Samuelson to have all the roots of a cubic polynomial inside the unit circle in the complex plane. The same conditions have been considered by many authors, e.g. in Okuguchi and Irie (1990), Gandolfo (2009) and Farebrother (2012). In short, let us recall the problem of the roots of a cubic polynomial

$$
P(x)=x^{3}+a_{1} x^{2}+a_{2} x+a_{3}
$$

where, without loss of generality, it is assumed that the first coefficient is given by $a_{0}=1$. The expressions of the four conditions, as given in Gandolfo (2009), are as follows:

$$
\begin{aligned}
\text { (i) } 1+a_{1}+a_{2}+a_{3} & >0 \\
\text { (ii) } 1-a_{1}+a_{2}-a_{3} & >0 \\
\text { (iii) } 1-a_{2}-a_{3}^{2}+a_{1} a_{3} & >0 \\
\text { (iv) } 3+a_{1}-a_{2}-3 a_{3} & >0
\end{aligned}
$$

Moreover, in a recent paper by Lines et al. (2019), which is useful in the applied context, it is shown that the last condition (condition (iv) above) is never violated when the parameters are varied from the region in which all the roots are inside the unit circle. Indeed, these results are quite robust, and our goal in this work is to extend these considerations, proving useful stability conditions and results on the associated bifurcations. In fact, we will comment on the codimension-1 bifurcations as well as the bifurcations of higher codimensions.

We will see that the last condition, (iv), can be considered equivalent to the following one:

$$
(i v)^{\prime}\left|a_{3}\right|<1
$$

meaning that there are always four conditions to check, but these are simplified in their application. In fact, just one condition is enough to state whether at least one root lies not inside the unit circle, which occurs for $\left|a_{3}\right| \geq 1$. For $\left|a_{3}\right|<1$ we can neglect this last condition and consider only the three remaining conditions, $(i)-(i i)-(i i i)$, in order to determine the stability region and the kinds of bifurcation that occur when some border of the region is crossed, with real or complex conjugate eigenvalues. We prove the following theorems.

Theorem $A$ (stability region). Consider a polynomial $P(x)=x^{3}+a_{1} x^{2}+a_{2} x+a_{3}$. All the roots of $P(x)$ are inside the unit circle iff the following conditions hold:

(i) $1+a_{1}+a_{2}+a_{3}>0$

(ii) $1-a_{1}+a_{2}-a_{3}>0$

(iii) $1-a_{2}-a_{3}^{2}+a_{1} a_{3}>0$,

$(i v)^{\prime}\left|a_{3}\right|<1$.

Consider the discriminant

$$
\mathcal{D}=4 a_{2}^{3}-a_{1}^{2} a_{2}^{2}+4 a_{1}^{3} a_{3}-18 a_{1} a_{2} a_{3}+27 a_{3}^{2} .
$$

For $\mathcal{D}<0$, three real roots exist, two real roots merge at $\mathcal{D}=0$, and for $\mathcal{D}>0$, there are two complex conjugate roots and a real one.

For the bifurcation conditions, let us consider a three-dimensional map and the Jacobian matrix evaluated at a fixed point. For the attractivity of the fixed point, a sufficient condition is that all eigenvalues are inside the unit circle in the complex plane. Assume that the coefficients of the characteristic polynomial are functions of a vector $\eta$ of parameters; here, too, we can assume the first coefficient is equal to 1. A bifurcation 
leading a fixed point from attracting to repelling can occur via at least one of the following equations (which give the boundaries of the stability region in the parameter space), at a bifurcation value that we call $\eta=\eta_{0}$ :

$$
\begin{gathered}
r_{1}\left(\eta_{0}\right): 1+a_{1}\left(\eta_{0}\right)+a_{2}\left(\eta_{0}\right)+a_{3}\left(\eta_{0}\right)=0 \\
r_{2}\left(\eta_{0}\right): 1-a_{1}\left(\eta_{0}\right)+a_{2}\left(\eta_{0}\right)-a_{3}\left(\eta_{0}\right)=0 \\
r_{3}\left(\eta_{0}\right): 1-a_{2}\left(\eta_{0}\right)-a_{3}\left(\eta_{0}\right)^{2}+a_{1}\left(\eta_{0}\right) a_{3}\left(\eta_{0}\right)=0
\end{gathered}
$$

The following results characterize the bifurcations.

Theorem $B$ (codimension-1 bifurcation points). Let $P(\lambda)=\lambda^{3}+a_{1}(\eta) \lambda^{2}+a_{2}(\eta) \lambda+a_{3}(\eta)$. Consider the vector parameter $\eta$ such that $\left|a_{3}(\eta)\right|<1$ and assume that the conditions in Theorem A are satisfied. As the parameter $\eta$ varies, let $\eta_{0}$ be a bifurcation value that always satisfies $\left|a_{3}\left(\eta_{0}\right)\right|<1$ and that is associated with parameters belonging to the border of the stability region. Assume that only one of the conditions in (5), (6) and (7) holds, i.e. only one of the three conditions (i), (ii) and (iii) is violated. We have that:

- if $r_{1}\left(\eta_{0}\right)$ holds, then one root is $\xi_{1}=+1$. Moreover, $a_{2}\left(\eta_{0}\right) \neq-1$ and

-if $1+a_{2}\left(\eta_{0}\right)>0$ and $\left(1-2 \sqrt{1+a_{2}\left(\eta_{0}\right)}\right)<a_{1}\left(\eta_{0}\right)<\left(1+2 \sqrt{1+a_{2}\left(\eta_{0}\right)}\right)$, then there are two complex conjugate roots $\xi_{2,3}=\alpha \pm i \beta$, where $\alpha=-\frac{1+a_{1}\left(\eta_{0}\right)}{2}, \beta=\sqrt{-a_{3}\left(\eta_{0}\right)-\alpha^{2}}$,

-if $1+a_{2}\left(\eta_{0}\right)>0$ and $a_{1}\left(\eta_{0}\right)=\left(1-2 \sqrt{1+a_{2}}\right)$ or $a_{1}\left(\eta_{0}\right)=\left(1+2 \sqrt{1+a_{2}\left(\eta_{0}\right)}\right)$, then there are two roots $\xi_{2}=\xi_{3}=-\frac{a_{1}\left(\eta_{0}\right)+1}{2}$,

-if $1+a_{2}\left(\eta_{0}\right)<0$, then there are two distinct real roots given by:

$$
\xi_{2,3}=\frac{1}{2}\left(-\left(a_{1}\left(\eta_{0}\right)+1\right) \pm \sqrt{\left(a_{1}\left(\eta_{0}\right)+1\right)^{2}+4 a_{3}\left(\eta_{0}\right)}\right) ;
$$

- if $r_{2}\left(\eta_{0}\right)$ holds, then one root is $\xi_{1}=-1$. Moreover, $a_{2}\left(\eta_{0}\right) \neq-1$ and

-if $1+a_{2}\left(\eta_{0}\right)>0$ and $\left(-1-2 \sqrt{1+a_{2}\left(\eta_{0}\right)}\right)<a_{1}\left(\eta_{0}\right)<\left(-1+2 \sqrt{1+a_{2}\left(\eta_{0}\right)}\right)$, then there are two complex conjugate roots $\xi_{2,3}=\alpha \pm i \beta$, where $\alpha=\frac{1-a_{1}\left(\eta_{0}\right)}{2}, \beta=\sqrt{a_{3}\left(\eta_{0}\right)-\alpha^{2}}$,

-if $1+a_{2}\left(\eta_{0}\right)>0$ and $a_{1}\left(\eta_{0}\right)=\left(-1-2 \sqrt{1+a_{2}\left(\eta_{0}\right)}\right)$ or $a_{1}\left(\eta_{0}\right)=\left(-1+2 \sqrt{1+a_{2}\left(\eta_{0}\right)}\right)$, then $\xi_{2}=$ $\xi_{3}=\frac{1-a_{1}\left(\eta_{0}\right)}{2}$,

-if $1+a_{2}\left(\eta_{0}\right)>0$, then there are two distinct real roots given by:

$$
\xi_{2,3}=\frac{1}{2}\left(\left(1-a_{1}\left(\eta_{0}\right)\right) \pm \sqrt{\left(1-a_{1}\left(\eta_{0}\right)\right)^{2}-4 a_{3}\left(\eta_{0}\right)}\right) ;
$$

- if $r_{3}\left(\eta_{0}\right)$ holds, then a pair of complex conjugate roots $\xi_{2,3}$ has modulus equal to 1 , and the real root is given by $\xi_{1}=-a_{3}\left(\eta_{0}\right)$.

Theorem $C$ (codimension-2 bifurcation points). Let $P(\lambda)=\lambda^{3}+a_{1}(\eta) \lambda^{2}+a_{2}(\eta) \lambda+a_{3}(\eta)$. Consider the vector parameter $\eta$ such that $\left|a_{3}(\eta)\right|<1$ and assume that the conditions in Theorem A are satisfied. As the parameter $\eta$ varies, let $\eta_{0}$ be a bifurcation value that always satisfies $\left|a_{3}\left(\eta_{0}\right)\right|<1$ and that is associated with parameters belonging to the border of the stability region. Assume that two, and only two, of the conditions in (5), (6) and (7) hold, i.e. two of the three conditions (i), (ii) and (iii) are violated. We have that:

- if $\eta_{0}$ is such that $r_{1}\left(\eta_{0}\right)$ and $r_{2}\left(\eta_{0}\right)$ hold, then the three roots are $\xi_{1}=+1, \xi_{2}=-1$ and $\xi_{3}=-a_{1}\left(\eta_{0}\right)=$ $a_{3}\left(\eta_{0}\right)$,

- if $\eta_{0}$ is such that $r_{1}\left(\eta_{0}\right)$ and $r_{3}\left(\eta_{0}\right)$ hold, then the three roots are $\xi_{1}=\xi_{2}=+1$ and $\xi_{3}=-a_{3}\left(\eta_{0}\right)$,

- if $\eta_{0}$ is such that $r_{2}\left(\eta_{0}\right)$ and $r_{3}\left(\eta_{0}\right)$ hold, then the three roots are $\xi_{1}=\xi_{2}=-1$ and $\xi_{3}=-a_{3}\left(\eta_{0}\right)$. 
Theorem $D$ (codimension-3 bifurcation points). Let $P(\lambda)=\lambda^{3}+a_{1}(\eta) \lambda^{2}+a_{2}(\eta) \lambda+a_{3}(\eta)$. Crossing the stability region at a vector parameter $\eta_{0}$ via $a_{3}\left(\eta_{0}\right)=+1$ or $a_{3}\left(\eta_{0}\right)=-1$ from inside to outside, the parameter point goes from the stability region to the instability region through a codimension-3 bifurcation.

- Let $a_{3}\left(\eta_{0}\right)=+1$. Then the closure of the stability region reduces to the set connecting the two points $\left(a_{1}\left(\eta_{0}\right), a_{2}\left(\eta_{0}\right), a_{3}\left(\eta_{0}\right)\right)=(-1,-1,+1)$ and $\left(a_{1}\left(\eta_{0}\right), a_{2}\left(\eta_{0}\right), a_{3}\left(\eta_{0}\right)\right)=(3,3,1)$, belonging to $a_{1}(\eta)=a_{2}(\eta)$ and $a_{3}(\eta)=1$. Crossing through the point $(-1,-1,+1)$, the eigenvalues are $\left(\xi_{1}, \xi_{2}, \xi_{3}\right)=$ $(-1,+1,+1)$; crossing through the point $(3,3,1)$ the eigenvalues are $\left(\xi_{1}, \xi_{2}, \xi_{3}\right)=(-1,-1,-1)$; crossing through the point $(a, a, 1)$, the eigenvalues are $\left(\xi_{1}, \xi_{2}, \xi_{3}\right)=\left(-1, \xi_{2}, \xi_{3}\right)$, with $\xi_{2}$ and $\xi_{3}$ complex conjugate in modulus equal to +1 and given by $\xi_{2,3}=\alpha \pm i \beta$, where $\alpha=\frac{1-a_{1}\left(\eta_{0}\right)}{2}, \beta=\sqrt{1-\alpha^{2}}$.

- Let $a_{3}\left(\eta_{0}\right)=-1$. Then the closure of the stability region reduces to the set connecting the two points $\left(a_{1}\left(\eta_{0}\right), a_{2}\left(\eta_{0}\right), a_{3}\left(\eta_{0}\right)\right)=(+1,-1,-1)$ and $\left(a_{1}\left(\eta_{0}\right), a_{2}\left(\eta_{0}\right), a_{3}\left(\eta_{0}\right)\right)=(-3,3,-1)$, belonging to $a_{2}(\eta)=$ $-a_{1}(\eta)$ and $a_{3}(\eta)=-1$. Crossing through the point $(+1,-1,-1)$, the eigenvalues are $\left(\xi_{1}, \xi_{2}, \xi_{3}\right)=$ $(-1,-1,+1)$; crossing through the point $(-3,3,-1)$, the eigenvalues are $\left(\xi_{1}, \xi_{2}, \xi_{3}\right)=(+1,+1,+1)$; crossing through the point $(a,-a,-1)$, the eigenvalues are $\left(\xi_{1}, \xi_{2}, \xi_{3}\right)=\left(+1, \xi_{2}, \xi_{3}\right)$, with $\xi_{2}$ and $\xi_{3}$ complex conjugate in modulus equal to +1 and given by $\xi_{2,3}=\alpha \pm i \beta$, where $\alpha=-\frac{1+a_{1}\left(\eta_{0}\right)}{2}, \beta=$ $\sqrt{1-\alpha^{2}}$.

A few more comments are in order.

- It is worth noting that conditions $(i v)$ and $(i v)^{\prime}$ - despite being equivalent in order to guarantee the fixed point's stability - are not equivalent in terms of the classification of the bifurcations related to the points on the boundaries of the stability region. In fact, as we can see from Theorem $D$, to achieve this goal only condition $(i v)^{\prime}$ is suitable, while Theorems $B$ and $C$ involve neither condition $(i v)$ nor $(i v)^{\prime}$.

- In a smooth nonlinear system, considering the Taylor expansion in a fixed point, not only the firstorder terms, which are related to the characteristic polynomial, are used, but also the higher-order terms, in order to further characterize the codimension- 1 bifurcations. This occurs by using the center manifold theorem of Hartman and Grobman (see, e.g. Guckenheimer and Holmes (2002), Kuznetsov (2004)). A bifurcation related to one eigenvalue equal to +1 can be associated with a fold, transcritical or pitchfork (subcritical or supercritical) bifurcation; the bifurcation related to one eigenvalue equal to -1 can be associated with a subcritical or supercritical flip bifurcation; the bifurcation related to a pair of complex eigenvalues may be a supercritical or subcritical Neimark-Sacker bifurcation, so that a Chenciner point associated with this transition may exist on the bifurcation set corresponding to $a_{3}\left(\eta_{0}\right)$ constant. A set of global bifurcations may issue from a Chenciner point. This is associated with codimension-2 bifurcations, due to the merging of two invariant sets of different nature, and related to homoclinic tangles (see, e.g. Gaunersdorfer et al. (2008)).

- We also mention that, for a cycle of period $k$ of a map, our analysis applies to a fixed point of the $k$-iterate of the map, and its Taylor expansion evaluated in one of the $k$ periodic points.

- We abstain from commenting on bifurcations of codimension-2 and -3 because these may lead to many different outcomes that have not yet been well studied even in the case of two-dimensional maps. Examples of the rich variety of outcomes that may occur at codimension-2 points in the two-dimensional case are given in Agliari et al. (2005).

- Finally, note that our results may also be applied to higher-order systems, provided that the characteristic polynomial may be factorized such that only a third-degree polynomial needs to be analyzed. See Lines and Westerhoff (2010), Tuinstra et al. (2014) and Schmitt and Westerhoff (2019) for examples in that direction.

To show how useful our mathematical insights are for applied research, we develop a simple housing market model in which homebuyers rely on a mix of nonlinear expectation rules to predict the development of house prices. Among other things, we show that the model's fundamental steady state may become 
unstable due to a Neimark-Sacker, pitchfork or flip bifurcation. It should be noted that a Neimark-Sacker bifurcation scenario is highly relevant to explain the boom-bust nature of real housing markets. This also holds for the pitchfork bifurcation scenario, as many real housing markets experience sustained periods of overvaluation or undervaluation. Clearly, proving analytically under which conditions economic systems may become unstable is obviously of utmost importance.

After this introduction, in Sec.2 we prove the aforementioned theorems. Sec.3 is devoted to developing our application to a system representing a housing market model, and Sec. 4 concludes.

\section{Proof of the theorems}

\subsection{Proof of Theorem $A$ (stability region)}

Since we know the four necessary and sufficient conditions given in (2) (e.g. Gandolfo (2009)), to prove our statement it suffices to show that these conditions hold iff conditions $(i)-(i i)-(i i i)-(i v)^{\prime}$ are satisfied. We therefore consider the conditions in (2) and identify the region in the parameter space determined by the coefficients $\left(a_{1}, a_{2}, a_{3}\right)$ in which these hold. To this end, we consider the surfaces representing the borders of the region, defining the closed sets as follows:

$$
\begin{array}{ll}
\overline{(i)} & a_{2} \geq-1-a_{1}-a_{3} \\
\overline{(i i)} & a_{2} \geq-1+a_{1}+a_{3} \\
\overline{(i i i)} & a_{2} \leq 1-a_{3}^{2}+a_{1} a_{3} \\
\overline{(i v)} & a_{2} \leq 3-3 a_{3}+a_{1}
\end{array}
$$

for which the equality determines the borders:

$$
\begin{array}{ll}
\left(r_{1}\right) & a_{2}=-1-a_{1}-a_{3} \\
\left(r_{2}\right) & a_{2}=-1+a_{1}+a_{3} \\
\left(r_{3}\right) & a_{2}=1-a_{3}^{2}+a_{1} a_{3} \\
\left(r_{4}\right) & a_{2}=3-3 a_{3}+a_{1}
\end{array}
$$

Let us assume that the coefficient $a_{3}$ is fixed, so that the borders are given by straight lines in the $\left(a_{1}, a_{2}\right)$ plane. Note that the straight lines satisfy the following properties:

(a) $\left(r_{1}\right)$ has a constant slope equal to -1 ,

(b) $\left(r_{2}\right)$ and $\left(r_{4}\right)$ have the same constant slope equal to 1 (i.e. the straight lines are parallel),

(c) $\left(r_{1}\right)$ and $\left(r_{4}\right)$ intersect at the point $\left(\alpha_{1}, \alpha_{2}\right)=R_{1,3,4}:=\left(a_{3}-2,1-2 a_{3}\right)$, which also belongs to the straight line $\left(r_{3}\right)$, i.e. these three lines intersect at the same point,

(d) $\left(r_{2}\right)$ and $\left(r_{1}\right)$ intersect orthogonally at the point $\left(\beta_{1}, \beta_{2}\right)=R_{1,2}:=\left(-a_{3},-1\right)$,

(e) $\left(r_{2}\right)$ and $\left(r_{3}\right)$ intersect, for $a_{3} \neq 1$, at the point $\left(\gamma_{1}, \gamma_{2}\right)=R_{2,3}:=\left(\frac{2-a_{3}-a_{3}^{2}}{1-a_{3}}, \frac{1+a_{3}-2 a_{3}^{2}}{1-a_{3}}\right)$; for $a_{3}=1$, the point becomes $\left(\gamma_{1}, \gamma_{2}\right)=R_{2,3}=(3,3)$.

A complete picture can be obtained by commenting separately on the three cases $a_{3}=0, a_{3}>0$ and $a_{3}<0$.

- Consider $a_{3}=0$. Then the straight lines $\left(r_{3}\right)$ and $\left(r_{4}\right)$ are the same, and the conditions reduce to the first three given in (2). This means that, in the $\left(a_{1}, a_{2}\right)$ plane, the region in which these conditions are satisfied is the stability triangle of the well-known two-dimensional case (see the gray region in Fig.1(a)), bounded by the first three lines. In fact, the cubic polynomial becomes $P(x)=x\left(x^{2}+a_{1} x+a_{2}\right)$, so that one root is $x=0$, and the two other roots are inside the unit circle when the usual conditions for $2 \mathrm{D}$ maps are satified. Thus, if $a_{3}=0$, then the conditions in $(2)$ hold iff $(i)-(i i)-(i i i)-(i v)^{\prime}$ hold.

- Consider $a_{3}>0$. First, for $0<a_{3}<1$, the slope of the straight line $\left(r_{3}\right)$ is positive and smaller than 1 (i.e. smaller than the slope of the lines $\left(r_{2}\right)$ and $\left(r_{4}\right)$ ), and it holds that $\alpha_{1}<\beta_{1}$ and $\alpha_{2}>\beta_{2}$. This means that, coupled with properties $(c)$ and $(d)$, the first three straight lines determine a bounded region. From property $(b)$ it follows that $\left(r_{4}\right)$ intersects the boundary at the corner point $R_{1,3,4}$, which is outside the open region bounded by the first three lines, and the open region belongs to the half-plane in which condition $(i v)$ is always satisfied (see Fig.1(b)). 
Second, for $a_{3}=1$, the three lines $\left(r_{2}\right),\left(r_{3}\right)$ and $\left(r_{4}\right)$ merge together into the line $a_{2}=a_{1}$, which is orthogonal to $\left(r_{1}\right)$. This means that there is no open region in which all conditions are satified, and the closed region reduces to a segment on the line $a_{2}=a_{1}$, connecting the point $\left(a_{1}, a_{2}\right)=R_{1,2}=(-1,-1)$ with the point in which $\left(r_{2}\right)$ intersects $\left(r_{3}\right)$, given by $R_{2,3}=(3,3)$.

Third, for $a_{3}>1$, the slope of the straight line $\left(r_{3}\right)$ is larger than 1 (i.e. larger than the slope of the lines $\left(r_{2}\right)$ and $\left.\left(r_{4}\right)\right)$, and it holds that $\alpha_{1}>\beta_{1}$ and $\alpha_{2}<\beta_{2}$. This means that the first three lines lead to an unbounded region in which the first three conditions hold (see the gray region in Fig.1(c)). The line $\left(r_{4}\right)$ intersects the closed region at the corner point $R_{1,3,4}$, but now the unbounded region belongs to the half-plane in which condition (iv) is not satisfied (nor is condition $(i v)^{\prime}$ ). Thus, the gray region is not a suitable region, and the role of the fourth condition is exactly to say that, in such a region, it is not true that all roots are inside the unit circle.
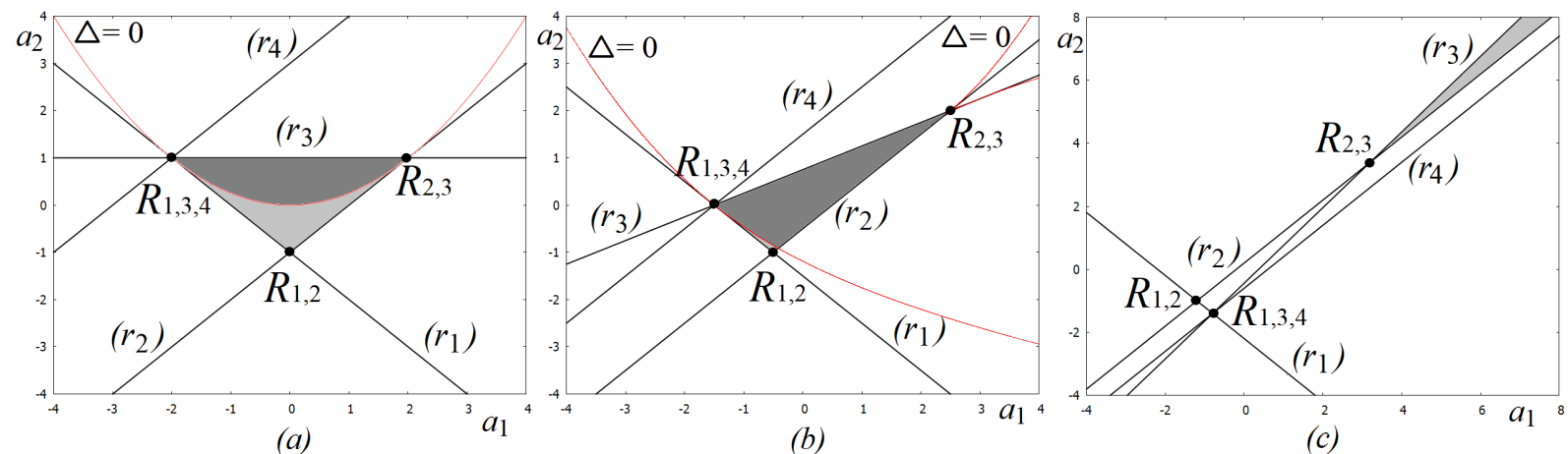

Fig.1 In (a) and (b), examples of stability regions are shown in gray tonalities. The dark gray region denotes the existence of a pair of complex conjugate roots, in (a) at $a_{3}=0$ and in (b) at $a_{3}=0.5$. In (c) at $a_{3}=1.2$, in the gray region, the conditions $(i),(i i)$ and (iii) are satisfied, but neither $(i v)$ nor $(i v)^{\prime}$.

- Consider $a_{3}<0$. The reasoning is similar to the previous one. First, for $-1<a_{3}<0$, the slope of the straight line $\left(r_{3}\right)$ is negative and larger than -1 (i.e. larger than the slope of the line $\left(r_{1}\right)$ ), and it holds that $\alpha_{1}<\beta_{1}$ and $\alpha_{2}>\beta_{2}$. This means that, coupled with properties $(c)$ and $(d)$, the first three straight lines determine a bounded region. From property $(b)$ it follows that line $\left(r_{4}\right)$ intersects the boundary at the corner point $R_{1,3,4}$, which is outside the open region bounded by the first three lines, and the open region belongs to the half-plane in which condition (iv) is always satisfied (see Fig.2(a)).

Second, for $a_{3}=-1$, the lines $\left(r_{1}\right)$ and $\left(r_{3}\right)$ merge together into the line $a_{2}=-a_{1}$ (and then switch). This means that there is no open region in which all conditions are satisfied, and the closed region reduces to the segment connecting the two points $\left(\alpha_{1}, \alpha_{2}\right)=R_{1,3,4}:=(-3,3)$ and $\left(\beta_{1}, \beta_{2}\right)=R_{1,2}:=(1,-1)$ (see Fig.2(b) for $a_{3}$ close to -1 ).
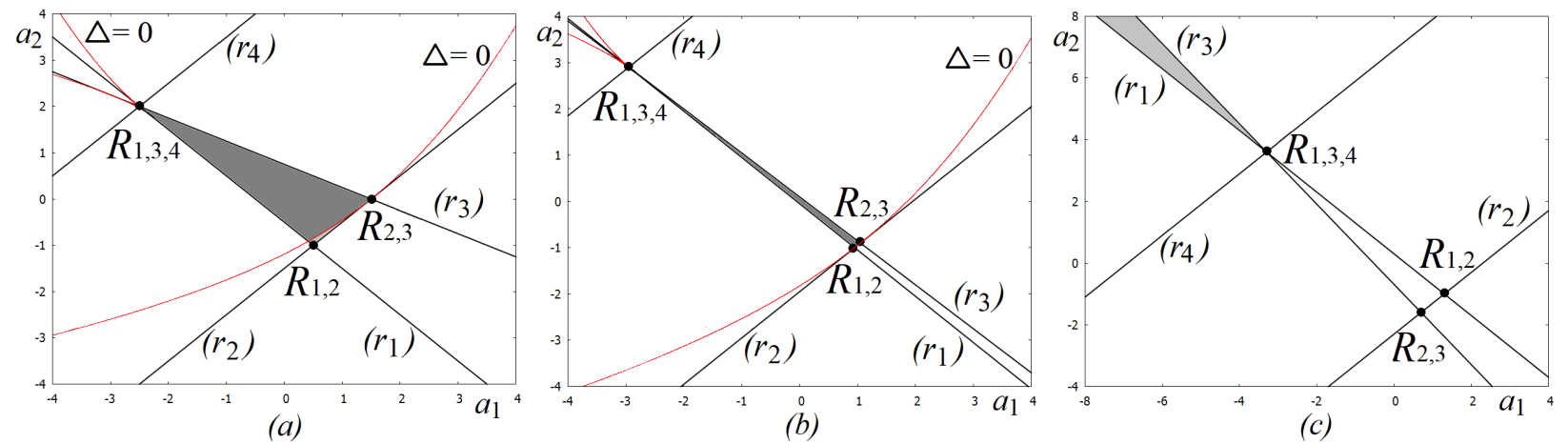

Fig.2 In (a) and (b), examples of stability regions are shown in gray tonalities. The dark gray region denotes the existence of a pair of complex conjugate roots, in (a) at $a_{3}=-0.5$ and in (b) at $a_{3}=-0.95$. In (c) at $a_{3}=-1.3$, in the gray region, the conditions $(i),(i i)$ and (iii) are satisfied, but neither $(i v)$ nor $(i v)^{\prime}$.

Third, for $a_{3}<-1$, the slope of the straight line $\left(r_{3}\right)$ is smaller than -1 (i.e. smaller than the slope of the line $\left(r_{1}\right)$ ). This means that the first three lines lead to an unbounded region in which the first three 
conditions hold (see the gray region in Fig.2(c)). The line $\left(r_{4}\right)$ intersects the closed region at the corner point $R_{1,3,4}$, but now the unbounded region belongs to the half-plane in which condition $(i v)$ is not satisfied (nor is condition $\left.(i v)^{\prime}\right)$. Thus, the gray region is not a suitable region, and the role of the fourth condition is exactly to say that, in such a region, it is not true that all the roots are inside the unit circle.

Combining the above results, all conditions in $(2)$ hold iff $(i)-(i i)-(i i i)-(i v)^{\prime}$ hold.

We now proceed to determine the types of roots that exist. We know that one root is always real, and the other roots may be real or complex. We can determine when two roots change from real to complex conjugate because this switch corresponds to the merging of two real roots (then entering the complex plane). More precisely, the transition of the eigenvalues from all real roots to one real root and a pair of complex conjugate roots is determined by the two following conditions:

$$
\begin{aligned}
P(x) & =x^{3}+a_{1} x^{2}+a_{2} x+a_{3}=0 \\
P^{\prime}(x) & =3 x^{2}+2 a_{1} x+a_{2}=0
\end{aligned}
$$

However, it is inappropriate to solve the system in this generic form. With the change of variable $x=y-\frac{a_{1}}{3}$, we get the cubic equation

$$
f(y)=y^{3}+p y+q
$$

where

$$
p=a_{2}-\frac{a_{1}^{2}}{3}, \quad q=a_{3}+2 \frac{a_{1}^{3}}{27}-\frac{a_{1} a_{2}}{3}
$$

The two conditions in (12) then become

$$
\begin{aligned}
f(y) & =y^{3}+p y+q=0 \\
f^{\prime}(y) & =3 y^{2}+p=0
\end{aligned}
$$

which can be solved explicitly giving

$$
\frac{q^{2}}{4}+\frac{p^{3}}{27}=0
$$

where $\mathcal{D}=\frac{q^{2}}{4}+\frac{p^{3}}{27}$ is usually called the discriminant. Consequently, the condition to have one unique real root and two complex conjugate ones is to have a positive discriminant, i.e. $\mathcal{D}>0$; two roots merge at $\mathcal{D}=0$, and for $\mathcal{D}<0$, three real roots exist. Substituting via (13) and simplifying, the condition in (14) leads to the equation in (4).

Consider the three-dimensional space $\left(a_{1}, a_{2}, a_{3}\right)$ depicted in Fig.3.

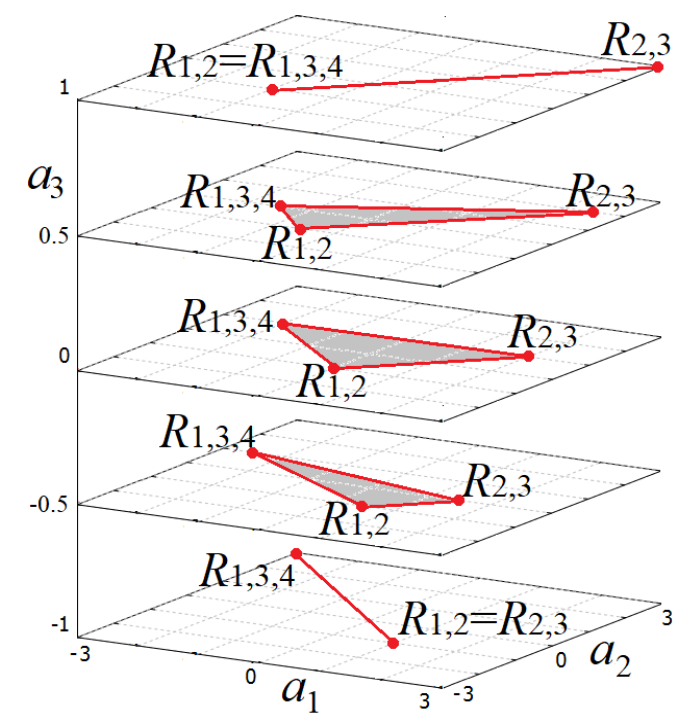

Fig.3 Sections of the stability volume, evidencing the boundaries related to bifurcations of codimension-1,

-2 and -3 . Bifurcations of codimension-3 occur only for $a_{3}=1$ and $a_{3}=-1$. 
Theorem A provides us with the complete volume of parameters, ensuring that all the eigenvalues are inside the unit circle, thereby identifying the stability region of a fixed point and its possible bifurcations. At each constant value $a_{3}=k$, we have the set described above. This means that, putting everything together, we have a volume in the space $\left(a_{1}, a_{2}, a_{3}\right)$ bounded by three surfaces that are planes intersecting $a_{3}=k$ for $|k|<1$ in the given triangle; for $a_{3}=1$, it reduces to a segment on the line $\left(a_{1}, a_{2}, 1\right)=(a, a, 1)$ with endpoints $(-1,-1,1)$ and $(3,3,1)$; and for $a_{3}=-1$ in a segment on the line $\left(a_{1}, a_{2},-1\right)=(a,-a,-1)$ with endpoints $(-3,3,-1)$ and $(1,-1,-1)$. Crossing the stability volume at a point $\left(a_{1}, a_{2}, k\right)$ with $|k|<1$, only codimension-1 and codimension-2 bifurcations can occur; a codimension-3 bifurcation occurs crossing the stability volume at a point $\left(a_{1}, a_{2}, k\right)$ with $|k|=1$. These bifurcations are discussed in the next subsections.

To achieve this goal, however, we first prove a Lemma and some corollaries that are used in the Theorems. The proof of the Lemma is reported in the Appendix, while the corollaries are an easy deduction.

Lemma 1 (roots of a cubic polynomial). Consider a cubic polynomial $P(x)=x^{3}+a_{1} x^{2}+a_{2} x+a_{3}$. Then one root, say $\xi_{1}$, must be real, and the other roots are as follows:

-if $\xi_{1}^{2}+a_{2}=0$, then $\xi_{2}=-\xi_{1}, \xi_{3}=-a_{1}$,

-if $\xi_{1}^{2}+a_{2}>0$ and

- $\left(\xi_{1}-2 \sqrt{\xi_{1}^{2}+a_{2}}\right)<a_{1}<\left(\xi_{1}+2 \sqrt{\xi_{1}^{2}+a_{2}}\right)$, then two complex conjugate roots exist, given by $\xi_{2,3}=$ $\alpha \pm i \beta$, where $\alpha=\frac{-\left(a_{1}+\xi_{1}\right)}{2}, \beta=\sqrt{\delta-\alpha^{2}}, \delta=-\frac{a_{3}}{\xi_{1}}$,

- $a_{1}=\left(\xi_{1}-2 \sqrt{\xi_{1}^{2}+a_{2}}\right)$ or $a_{1}=\left(\xi_{1}+2 \sqrt{\xi_{1}^{2}+a_{2}}\right)$, then $\xi_{2}=\xi_{3}=\frac{\tau}{2}=-\frac{a_{1}+\xi_{1}}{2}$.

In all other cases, there are two distinct real roots, given by:

$$
\xi_{2,3}=\frac{1}{2}(\tau \pm \sqrt{\Delta}), \text { where } \Delta=\tau^{2}-4 \delta, \tau=-\left(a_{1}+\xi_{1}\right), \delta=-\frac{a_{3}}{\xi_{1}}
$$

Corollary 1. Consider a cubic polynomial $P(x)=x^{3}+a_{1} x^{2}+a_{2} x+a_{3}$ with the real root $\xi_{1}=1$. The other roots are as follows:

-if $1+a_{2}=0$, then two real roots exist, given by $\xi_{2}=-1, \xi_{3}=-a_{1}$,

-if $1+a_{2}>0$ and

- $\left(1-2 \sqrt{1+a_{2}}\right)<a_{1}<\left(1+2 \sqrt{1+a_{2}}\right)$, then two complex conjugate roots exist, given by $\xi_{2,3}=\alpha \pm i \beta$, where $\alpha=-\frac{1+a_{1}}{2}, \beta=\sqrt{-a_{3}-\alpha^{2}}$,

- $a_{1}=\left(1-2 \sqrt{1+a_{2}}\right)$ or $a_{1}=\left(1+2 \sqrt{1+a_{2}}\right)$, then two real coincident roots exist, given by $\xi_{2}=\xi_{3}=$ $-\frac{a_{1}+1}{2}$.

In all other cases, there are two distinct real roots, given by:

$$
\xi_{2,3}=\frac{1}{2}\left(\tau \pm \sqrt{\tau^{2}+4 a_{3}}\right) \text {, where } \tau=-\left(a_{1}+1\right)
$$

Corollary 2. Consider a cubic polynomial $P(x)=x^{3}+a_{1} x^{2}+a_{2} x+a_{3}$ with the real root $\xi_{1}=-1$. The other roots are as follows:

-if $1+a_{2}=0$, then two real roots exist, given by $\xi_{2}=1, \xi_{3}=-a_{1}$,

-if $1+a_{2}>0$ and

- $\left(-1-2 \sqrt{1+a_{2}}\right)<a_{1}<\left(-1+2 \sqrt{1+a_{2}}\right)$, then two complex conjugate roots exist, given by $\xi_{2,3}=$ $\alpha \pm i \beta$, where $\alpha=\frac{1-a_{1}}{2}, \beta=\sqrt{a_{3}-\alpha^{2}}$,

- $a_{1}=\left(-1-2 \sqrt{1+a_{2}}\right)$ or $a_{1}=\left(-1+2 \sqrt{1+a_{2}}\right)$, then two real coincident roots exist, given by $\xi_{2}=$ $\xi_{3}=\frac{1-a_{1}}{2}$. 
In all other cases, there are two distinct real roots, given by:

$$
\xi_{2,3}=\frac{1}{2}\left(\tau \pm \sqrt{\tau^{2}-4 a_{3}}\right) \text {, where } \tau=1-a_{1}
$$

Corollary 3. Consider a cubic polynomial $P(x)=x^{3}+a_{1} x^{2}+a_{2} x+a_{3}$ with

$(j)$-two real roots given by $\xi_{1}=1$ and $\xi_{2}=-1$, then $\xi_{3}=-a_{1}=a_{3}$,

$(j j)$-two real roots given by $\xi_{1}=\xi_{2}=1$, then $\xi_{3}=-a_{3}$,

$(j j j)$-two real roots given by $\xi_{1}=\xi_{2}=-1$, then $\xi_{3}=-a_{3}$,

(jv)-two complex conjugate roots $\xi_{2,3}$ in modulus equal to 1 , then $\xi_{1}=-a_{3}$.

\subsection{Proof of Theorem $B$ (codimension-1 bifurcation points)}

For a polynomial of odd degree, there exists at least one real root. Moreover, the meaning of the coefficients in terms of the roots, say $P(\lambda)=\left(\lambda-\xi_{1}\right)\left(\lambda-\xi_{2}\right)\left(\lambda-\xi_{3}\right)$, is as follows:

$$
\begin{aligned}
& a_{1}(\eta)=-\left(\xi_{1}+\xi_{2}+\xi_{3}\right) \\
& a_{2}(\eta)=+\left(\xi_{1} \xi_{2}+\xi_{1} \xi_{3}+\xi_{2} \xi_{3}\right) \\
& a_{3}(\eta)=-\left(\xi_{1} \xi_{2} \xi_{3}\right)
\end{aligned}
$$

Since as $\lambda \rightarrow+\infty$ the value of $P(\lambda) \rightarrow+\infty$, and for $\lambda \rightarrow-\infty$ the value of $P(\lambda) \rightarrow-\infty$, either the polynomial has no critical points (no local extrema), or the critical points, say $\xi_{c 1} \leq \xi_{c 2}$, are such that when disjoint, $\xi_{c 1}$ is a local maximum and $\xi_{c 2}$ a local minimum.

It follows (from the shape of the polynomial) that, if all the roots are larger than -1 , then it must be $P(-1)<0$; for $P(-1)=0$ at least one root is -1 ; and when $P(-1)>0$, then there exists at least one real root smaller than -1 .

Reasoning similarly, from the shape of the polynomial we have that if all the roots are smaller than 1 , then it must be $P(1)>0$, for $P(1)=0$ at least one root is 1 , and when $P(1)<0$, then there exists at least one real root larger than 1.

In our case, $P(1)=1+a_{1}(\eta)+a_{2}(\eta)+a_{3}(\eta)$ and $P(-1)=-\left(1-a_{1}(\eta)+a_{2}(\eta)-a_{3}(\eta)\right)$. Thus, when the first two conditions in Theorem A given by

$$
\begin{aligned}
\text { (i) } P(1) & =1+a_{1}(\eta)+a_{2}(\eta)+a_{3}(\eta)>0 \\
\text { (ii) } P(-1) & =-\left(1-a_{1}(\eta)+a_{2}(\eta)-a_{3}(\eta)\right)<0
\end{aligned}
$$

are satisfied, we can state that no real root is outside to the interval $(-1,1)$.

At the bifurcation, if

$$
r_{1}\left(\eta_{0}\right)=0, \quad P(1)=1+a_{1}\left(\eta_{0}\right)+a_{2}\left(\eta_{0}\right)+a_{3}\left(\eta_{0}\right)=0
$$

then at least one root is equal to 1 , and from Corollary 1 we have explicitly all the other roots, real or complex.

Similarly, if

$$
r_{2}\left(\eta_{0}\right)=0, \quad P(-1)=-\left(1+a_{1}\left(\eta_{0}\right)+a_{2}\left(\eta_{0}\right)+a_{3}\left(\eta_{0}\right)\right)=0
$$

then at least one root is equal to -1 , and from Corollary 2 we have explicitly all the other roots, real or complex.

Now, assuming $\left|a_{3}\left(\eta_{0}\right)\right|=\left|\xi_{1} \xi_{2} \xi_{3}\right|<1$, then the third condition $r_{3}\left(\eta_{0}\right)=0$ is necessarily related to complex conjugate eigenvalues. That is, if we cross the border of the stability region when $(i)$ and $(i i)$ hold, then the real root is certainly in the interval $(-1,1)$, and the only possibility to have instability is via a pair of complex conjugate roots which become larger than 1 in modulus, and the boundary condition $r_{3}\left(\eta_{0}\right)=0$ corresponds to a pair of complex conjugate roots, say $\xi_{2}$ and $\xi_{3}$, equal to 1 in modulus. Note that, in such a case, at the condition $r_{3}\left(\eta_{0}\right)=0$, the determinant of the polynomial is still $\left|a_{3}\left(\eta_{0}\right)\right|<1$ because one real root is in the interval $(-1,1)$, and from Corollary $3-(j v)$ we have that $\xi_{3}=-a_{3}\left(\eta_{0}\right)$.

Some examples are reported in Fig.4, showing the graph of the polynomial at the codimension-1 bifurcation points labelled as $A, B$ and $C$, when condition $\left(r_{1}\right),\left(r_{3}\right)$ and $\left(r_{2}\right)$ holds, respectively. 

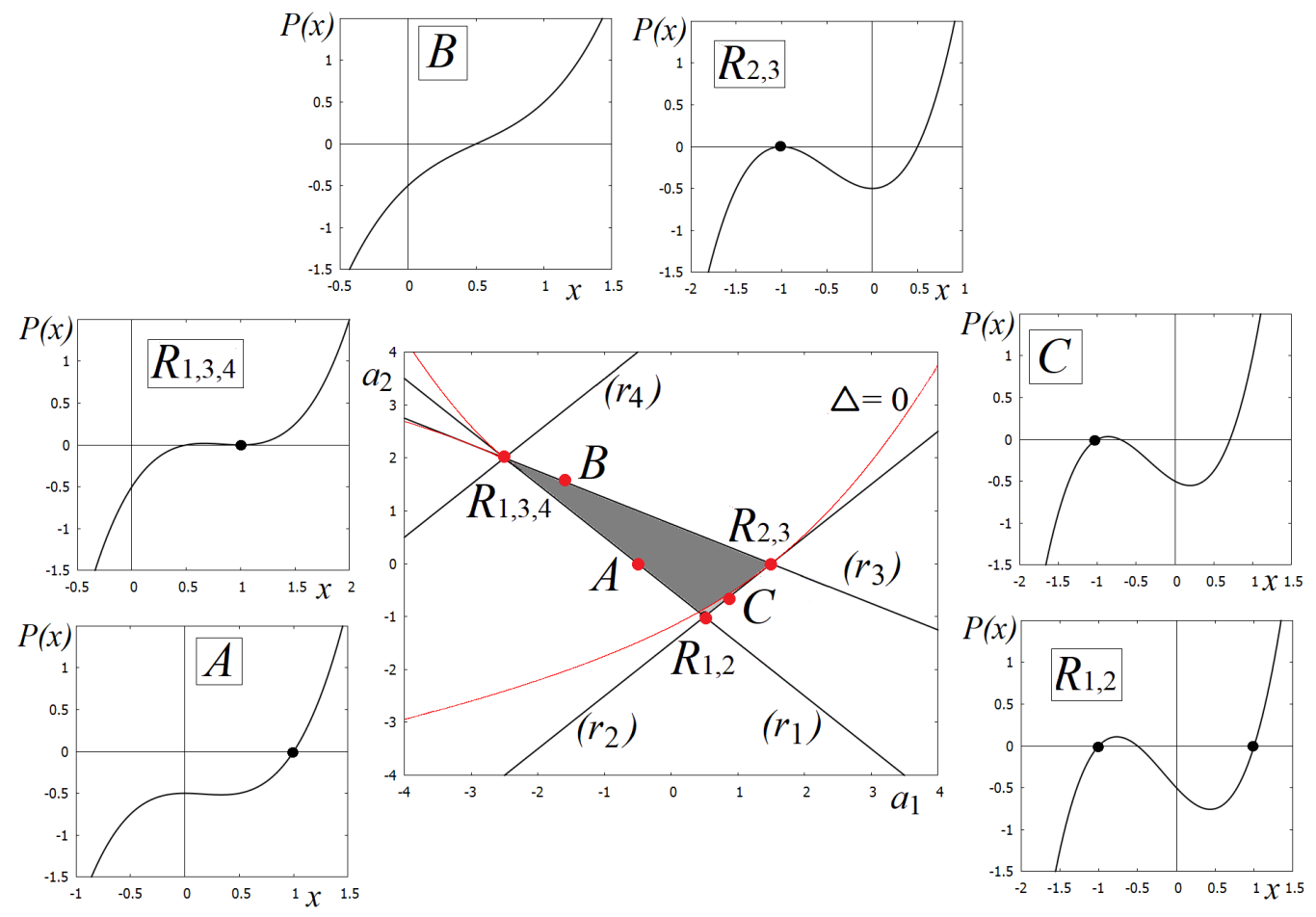

Fig.4 Qualitative representation of the polynomial at a number of bifurcation values, for $-1<a_{3}<0$. At points $A, B$ and $C$, a codimension- 1 bifurcation occurs. At points $R_{1,2}, R_{2,3}$ and $R_{1,3,4}$, a codimension-2

bifurcation occurs. A black dot denotes an eigenvalue -1 or +1 .

\subsection{Proof of Theorem $C$ (codimension-2 bifurcation points)}

If $\eta_{0}$ is such that $r_{1}\left(\eta_{0}\right)$ and $r_{2}\left(\eta_{0}\right)$ hold, then $P(1)=0$ and $P(-1)=0$ (i.e. the parameters are given by the point $\left(\beta_{1}, \beta_{2}\right)=R_{1,2}=\left(-a_{3}\left(\eta_{0}\right),-1\right)$, see proof of Theorem $\left.\mathrm{A}\right)$, so that one root is +1 and one root is -1 . The third root follows from Corollary $3-(j)$.

If $\eta_{0}$ is such that $r_{1}\left(\eta_{0}\right)$ and $r_{3}\left(\eta_{0}\right)$ hold, then $P(1)=0$. This means that one root is +1 , and the parameters are given by the point $\left(\alpha_{1}, \alpha_{2}\right)=R_{1,3,4}=\left(a_{3}\left(\eta_{0}\right)-2,1-2 a_{3}\left(\eta_{0}\right)\right)$, see proof of Theorem A. Thus, two complex conjugate roots must also exist. This is only possible if the two roots are equal to +1 . The third root follows from Corollary $3-(j j)$.

If $\eta_{0}$ is such that $r_{2}\left(\eta_{0}\right)$ and $r_{3}\left(\eta_{0}\right)$ hold, then $P(-1)=0$. This means that one root is -1 , and the parameters are given by the point defined $R_{2,3}$, see proof of Theorem A, for which two complex conjugate roots must exist. This is only possible if the two roots are equal to -1 . The third root follows from Corollary $3-(j j j)$.

Some examples are reported in Fig.4, showing the graph of the polynomial at the codimension-2 bifurcation points $R_{1,2}, R_{2,3}$, and $R_{1,3,4}$ for the case $-1<a_{3}<0$.

\subsection{Proof of Theorem $D$ (codimension-3 bifurcation points)}

The proof of Theorem A shows that, if $a_{3}=1$, then the closure of the stability region reduces to the segment belonging to the straight line $a_{2}=a_{1}$, connecting the two points $\left(a_{1}, a_{2}\right)=R_{1,2}=(-1,-1)$ with the point $\left(\gamma_{1}, \gamma_{2}\right)=R_{2,3}=(3,3)$, and the straight lines $r_{2}$ and $r_{3}$ merge. At the point $\left(a_{1}, a_{2}\right)=R_{1,2}=(-1,-1)$, one root is equal to -1 (from $P(-1)=0$ ) and one root is equal to +1 (from $P(1)=0$ ); and since the determinant is -1 , the third root must necessarily be equal to +1 , that is, $\left(\xi_{1}, \xi_{2}, \xi_{3}\right)=(-1,+1,+1)$. In the other extremum $\left(\gamma_{1}, \gamma_{2}\right)=R_{2,3}=(3,3)$, one root is equal to -1 and the other two roots are real, so that it must be $\left(\xi_{1}, \xi_{2}, \xi_{3}\right)=(-1,-1,-1)$. At a point inside the segment, one root is equal to -1 (from 
$P(-1)=0)$ and two roots are complex conjugate, equal to 1 in modulus, say $\left(\xi_{1}, \xi_{2}, \xi_{3}\right)=\left(-1, \xi_{2}, \xi_{3}\right)$, and from Corollary 2 the complex roots are given by $\xi_{2,3}=\alpha \pm i \beta$, where $\alpha=\frac{1-a_{1}}{2}, \beta=\sqrt{1-\alpha^{2}}$.

If $a_{3}=-1$, then the closure of the stability region reduces to the segment belonging to the straight line $a_{2}=-a_{1}$ connecting the two points $\left(\alpha_{1}, \alpha_{2}\right)=R_{1,3,4}=(-3,3)$ and the point $\left(\beta_{1}, \beta_{2}\right)=R_{1,2}=(1,-1)$; the straight lines $\left(r_{1}\right)$ and $\left(r_{3}\right)$ merge together, which means that one root is equal to +1 (from $P(1)=0$ ), and two more roots are equal to 1 in modulus (from $r_{3}(\eta)$ ). At a point inside that segment, the roots are complex conjugate, in modulus equal to 1 , say $\left(\xi_{1}, \xi_{2}, \xi_{3}\right)=\left(1, \xi_{2}, \xi_{3}\right)$, and from Corollary 1 the complex roots are given by $\xi_{2,3}=\alpha \pm i \beta$, where $\alpha=-\frac{1+a_{1}}{2}, \beta=\sqrt{1-\alpha^{2}}$. In contrast, the roots are real at the extrema. At the point $R_{1,3,4}=(-3,3)$, three real roots are equal to +1 , that is, $\left(\xi_{1}, \xi_{2}, \xi_{3}\right)=(+1,+1,+1)$, while at the point $\left(\beta_{1}, \beta_{2}\right)=R_{1,2}=(1,-1)$, one root is equal to -1 (from $\left.P(-1)=0\right)$ and one root is equal to +1 (from $P(1)=0$ ); and since the determinant is +1 , the third root must necessarily be equal to -1 , that is, $\left(\xi_{1}, \xi_{2}, \xi_{3}\right)=(-1,-1,+1)$.

Except for such points that cover all cases in which there are three roots equal to +1 in modulus, when the condition $\left|a_{3}\right|=1$ holds, the eigenvalues are already outside the unit circle. Such a transition is associated, for example, to one root $\xi$, one root $\frac{1}{\xi}$ and a third real root \pm 1 , or a pair of complex roots with modulus $M$ and a real root $\frac{1}{M}$; in all such cases, one root is already outside the unit circle.

Thus, going back to the parameter space that depends on a vector of other parameters $\left(a_{1}(\eta), a_{2}(\eta), a_{3}(\eta)\right)$, and a vector parameter $\eta_{0}$ at which $a_{3}\left(\eta_{0}\right)=+1$ or $a_{3}\left(\eta_{0}\right)=-1$, the statements in Theorem $D$ come soon. $\square$

Remark. It is worth noting that the two conditions $(i v)$ and $(i v)^{\prime}$, despite being equivalent in order to guarantee a fixed point's stability, are not equivalent in terms of the classification of the bifurcations related to the points on the boundary of the stability region. In fact, as we have shown in Theorem $C$, at the point denoted as $R_{1,3,4}$ in Theorem $A$, when $\left|a_{3}\left(\eta_{0}\right)\right|<1$ we have that three of the four conditions given in (2) are violated, but not condition $(i v)^{\prime}$, and at that point a codimension-2 bifurcation occurs. It is only when condition $(i v)^{\prime}$ is violated together with two of the first three conditions in (2) that a codimension-3 bifurcation occurs, as shown in Theorem $D$.

\section{A housing market model}

In this section, we apply some of our mathematical insights to a simple housing market model. Our housing market model is related to those by Schmitt and Westerhoff $(2019,2020)$, which, in turn, descend from the housing market models by Dieci and Westerhoff (2016) and Poterba (1984, 1991). For more work in this direction, see the inspiring survey by Dieci and He (2018).

\subsection{A simple housing market model}

There is a single price of housing paid by all (risk-neutral) homebuyers, obeying a standard intertemporal noarbitrage condition. Accordingly, the house price $P_{t}$ equals the discounted value of the next period's expected house price $E_{t}\left[P_{t+1}\right]$ plus (hypothetical) rent payments $R_{t}$, due at the end of the period. In mathematical terms, the house price in period $t$ is given by

$$
P_{t}=\frac{E_{t}\left[P_{t+1}\right]+R_{t}}{1+r+\delta}
$$

where $r>0$ stands for the risk-free interest rate, guaranteed by homebuyers' alternative investment option, say, via safe government bonds, and $0<\delta<1$ denotes the housing depreciation rate.

For the real part of our housing market model, we assume that the rent level decreases with the current stock of houses

$$
R_{t}=\alpha-\beta H_{t}
$$

with $\alpha>0, \beta>0$, and that the current housing stock evolves as

$$
H_{t}=I_{t}+(1-\delta) H_{t-1}
$$


where new housing construction depends positively on the past house price, i.e.

$$
I_{t}=\gamma P_{t-1}
$$

with $\gamma>0$. Note that new housing construction is consistent with the assumptions that house builders face a one-period production lag, maximize expected profits subject to a quadratic cost function, i.e. $C_{t}=\frac{1}{2 \gamma} I_{t}^{2}$, and form naïve expectations. Consequently, lower values of $\gamma$ imply higher building costs.

If homebuyers expect no further house price changes, the house price corresponds to the discounted value of future rent payments. More formally, for $P_{t}=E_{t}\left[P_{t+1}\right]$, we obtain from (18) that $P_{t}=\frac{R_{t}}{r+\delta}$. Straightforward computations, using (19)-(21), furthermore reveal that the model's long-run equilibrium house price, also called the fundamental house price, is given by $F=\frac{\alpha \delta}{(r+\delta) \delta+\beta \gamma}$. In reality, however, the house price rarely mirrors its fundamental value. As pointed out by Case and Shiller (2003) and Case et al. (2012), for instance, homebuyers' optimistic expectations have created sustained periods of overvaluation and significant bubbles in the past. Of course, sustained periods of undervaluation and severe crashes may occur if homebuyers turn pessimistic. In the economic literature (Dieci and He (2018)), two competing formulations have evolved to model such expectations, and we seek to integrate both of them in our model. Some models assume that homebuyers believe in trends, while others assume that homebuyers believe in the persistence of bull and bear markets. We thus formalize homebuyers' house price expectations by

$$
E_{t}\left[P_{t+1}\right]=P_{t-1}+\eta \arctan \left(\frac{\chi}{\eta}\left(P_{t-1}-P_{t-2}\right)\right)+\varepsilon \arctan \left(\frac{\phi}{\varepsilon}\left(P_{t-1}-F\right)\right)
$$

where $\eta=\frac{2 k}{\pi}>0, \chi>0, \varepsilon=\frac{2 \tau}{\pi}>0$ and $\phi>0$. Clearly, homebuyers become optimistic (pessimistic) if the house price increases (decreases) and/or if the house price climbs above (drops below) its fundamental value. Note that the S-shaped arctangent functions in (22), bounded in the intervals $(-k, k)$ and $(-\tau, \tau)$, reflect homebuyers' tendency to react more cautiously to larger price changes and to larger mispricing. However, the latter part of (22) may also imply that homebuyers form regressive expectations. More precisely, by setting $\phi<0$ and $\tau \leq 1$, we obtain a model in which homebuyers expect the house price to return towards its fundamental value.

\subsection{Analytical insights}

Introducing the auxiliary variable $X_{t}=P_{t-1}$ allows us to express our model by a 3D map $T$, given by:

$$
\left\{\begin{array}{l}
P_{t}=\frac{1}{1+r+\delta}\left[P_{t-1}+\eta \arctan \left(\frac{\chi}{\eta}\left(P_{t-1}-X_{t-1}\right)\right)+\varepsilon \arctan \left(\frac{\phi}{\varepsilon}\left(P_{t-1}-F\right)\right)+\alpha-\beta \gamma P_{t-1}-\beta(1-\delta) H_{t-1}\right] \\
X_{t}=P_{t-1} \\
H_{t}=\gamma P_{t-1}+(1-\delta) H_{t-1}
\end{array}\right.
$$

i.e. its dynamics is driven by the iteration of a three-dimensional nonlinear map.

By imposing the steady-state conditions $\bar{P}=P_{t}=P_{t-1}, \bar{X}=X_{t}=X_{t-1}$ and $\bar{H}=H_{t}=H_{t-1}$, we obtain the model's fundamental steady state $S=\left(\bar{P}, \bar{P}, \frac{\gamma}{\delta} \bar{P}\right)$, where $\bar{P}=F=\frac{\alpha \delta}{(r+\delta) \delta+\beta \gamma}$. It furthermore follows that $\bar{H}=\frac{\alpha \gamma}{(r+\delta) \delta+\beta \gamma}$ and $\bar{R}=\frac{(r+\delta) \delta \alpha}{(r+\delta) \delta+\beta \gamma}$. Accordingly, $\bar{P}, \bar{H}$ and $\bar{R}$ are solely determined by the model's real parameters. While homebuyers using expectation rule (22) make no prediction errors at the fundamental steady state, it should be noted that the model may possess further non-fundamental steady states where this is not the case. Numerical experiments, presented in the sequel, reveal that these non-fundamental steady states may be subject to the model's behavioral parameters.

The Jacobian matrix, evaluated at the fundamental steady state, is given by

$$
J(S)=\left(\begin{array}{ccc}
\frac{1-\beta \gamma+\chi+\phi}{1+r+\delta} & -\frac{\chi}{1+r+\delta} & -\frac{\beta(1-\delta)}{1+r+\delta} \\
1 & 0 & 0 \\
\gamma & 0 & 1-\delta
\end{array}\right)
$$

and leads to the characteristic polynomial

$$
\mathcal{P}(\lambda)=\lambda^{3}+a_{1} \lambda^{2}+a_{2} \lambda+a_{3}=0
$$


where

$$
a_{1}=\frac{\beta \gamma+\delta(r+\delta)-2-r-\chi-\phi}{1+r+\delta}, a_{2}=\frac{1+2 \chi+\phi-\delta(1+\chi+\phi)}{1+r+\delta}, a_{3}=-\frac{(1-\delta) \chi}{1+r+\delta}
$$

We can now apply Theorem A to study the stability of the model's fundamental steady state. From conditions $(i)-(i i)-(i i i)-(i v)^{\prime}$, we obtain the following conditions in terms of our model parameters:

$$
\begin{aligned}
& \left(i i^{\prime}\right) \quad \delta(r+\delta)+\beta \gamma<4+2 r+(2 \chi+\phi)(2-\delta) \\
& \left(i i i^{\prime}\right) \delta \chi+\frac{\beta \gamma \chi}{1+r+\delta-\chi}+\phi<\frac{2 r+\delta}{1-\delta} \\
& \left(i v^{\prime}\right)^{\prime} \quad \frac{1+r+\delta}{1-\delta}<\chi<\frac{1+r+\delta}{1-\delta}
\end{aligned}
$$

Since $\chi>0$, only the second inequality of $\left(i v^{\prime}\right)^{\prime}$ is of economic relevance. Moreover, note that the second term on the left-hand side of $\left(i i i^{\prime}\right)$ goes to infinity as $\chi$ approaches $1+r+\delta$. Hence, inequality $\left(i i i^{\prime}\right)$ will always be violated before inequality $\left(i v^{\prime}\right)^{\prime}$ becomes violated as $\chi$ increases. Let us next assume that the model's fundamental steady state is stable. According to Theorem B, a separate violation of inequalities $\left(i^{\prime}\right),\left(i i^{\prime}\right)$ and $\left(i i i^{\prime}\right)$, while the other two conditions hold, is then associated with a fold (or transcritical or pitchfork), flip and Neimark-Sacker bifurcation, respectively.

\subsection{Numerical insights}

Let us now turn to a numerical exploration of our model. To demonstrate that the model's fundamental steady state may indeed lose its stability via three different types of bifurcation, we introduce three parameter settings. While our focus rests on the evolution of the house price, we remark that the housing stock and the rent level always remain positive in our simulations.

Parameter setting 1: We observe a supercritical Neimark-Sacker bifurcation if we set $\alpha=20, \beta=0.1$, $\gamma=0.05, \delta=0.1, r=0.1, k=1, \phi=0.2, \tau=1$ and use $\chi$ as a bifurcation parameter, so that condition (iii $\left.i^{\prime}\right)$ becomes violated. Our analytical results predict that the model's fundamental steady state, characterized by $\bar{P}=80, \bar{H}=40$ and $\bar{R}=16$, becomes unstable due to a Neimark-Sacker bifurcation as parameter $\chi$ crosses the bifurcation value $\chi_{0}^{N S} \approx 1.02$. This bifurcation scenario is depicted in Fig.5(a). As we can see, our housing market model may produce endogenous boom-bust cycles if homebuyers extrapolate past house price changes sufficiently strongly. The empirical evidence presented in Dieci and He (2018) suggests that the dynamics of real housing markets is best characterized by oscillatory fluctuations, underlying the relevance of the Neimark-Sacker bifurcation scenario for the analysis of housing market dynamics. Interestingly, it is clear from $\left(i i i^{\prime}\right)$ that such a bifurcation scenario may also occur if homebuyers become more bullish (bearish) if the housing market is overvalued (undervalued), i.e. if parameter $\phi$ increases. In fact, cyclical housing market dynamics may even occur if homebuyers do not extrapolate past house price trends $(\chi=0)$, a rare outcome in this line of research. Economically, we can thus conclude that the emergence of endogenous boom-bust housing market dynamics is a quite robust phenomenon, as it may result from different specifications of homebuyers' expectation formation behavior.

Parameter setting 2: Recall that one eigenvalue equal to +1 may be associated with a fold bifurcation, a transcritical bifurcation or a pitchfork bifurcation. In our system we have observed that a pitchfork bifurcation occurs when condition $\left(i^{\prime}\right)$ becomes violated. To illustrate the pitchfork bifurcation scenario, we set $\alpha=20, \beta=0.1, \gamma=0.05, \delta=0.1, r=0.1, k=1, \chi=0.2, \tau=1$ and use $\phi$ as a bifurcation parameter. Now our analytical results predict that the model's fundamental steady state, again characterized by $\bar{P}=80$, $\bar{H}=40$ and $\bar{R}=16$, becomes unstable due to a pitchfork bifurcation as parameter $\phi$ increases above the bifurcation value $\phi_{0}^{\text {pitch }} \approx 0.25$. In Fig.5(b) we illustrate the corresponding numerical evidence. Depending on the initial conditions, homebuyers' expectations may lead to a permanent overvaluation or undervaluation of the housing market, shown in red and blue, respectively. This phenomenon is also reported in the housing market literature (Dieci and He (2018)). Note that a small amount of exogenous noise may create interesting attractor switching dynamics. For instance, the house price may fluctuate around the model's upper nonfundamental steady state for a while and then, apparently out of the blue, crash and fluctuate around its 
lower non-fundamental steady state. Note that such regime changes require that exogenous shocks push the system from one basin of attraction to another.

(a)

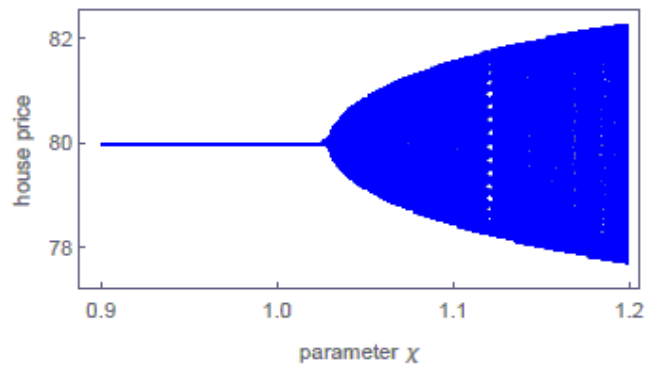

(b)

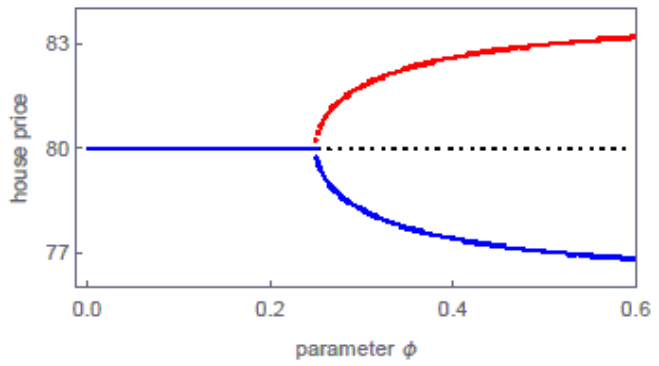

(c)

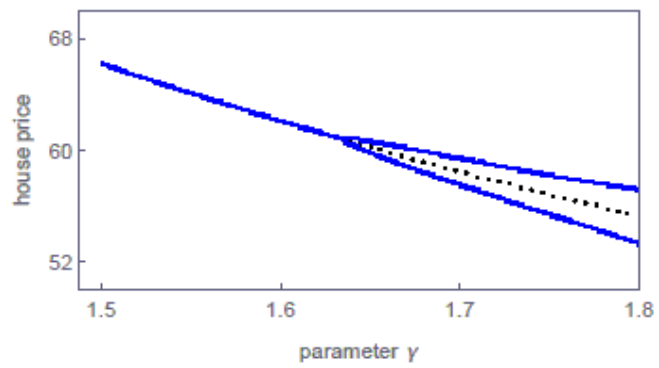

Fig. 5 Bifurcation routes of house prices. In (a) bifurcation diagram as a function of parameter $\chi$ (parameter setting 1). In (b) bifurcation diagram as a function of parameter $\phi$ (parameter setting 2 , two different sets of initial conditions). In (c) bifurcation diagram as a function of parameter $\gamma$ (parameter setting 3$)$.

Parameter setting 3: Although no evidence for a flip bifurcation scenario has been reported for real housing markets as yet, for completeness we also discuss this possibility. Let us set $\alpha=2000, \beta=2, \delta=0.1$, $r=0.1, \chi=0.1, k=1, \phi=-0.5, \tau=1$ and use $\gamma$ as a bifurcation parameter, so that condition $\left(i i^{\prime}\right)$ becomes violated. Accordingly, the model's fundamental steady state, given by $\bar{P}=\frac{200}{0.02+2 \gamma}, \bar{H}=\frac{2000 \gamma}{0.02+2 \gamma}$ and $\bar{R}=2000-\frac{4000}{0.02+2 \gamma}$, becomes unstable due to a flip bifurcation as parameter $\gamma$ exceeds the bifurcation value $\gamma_{0}^{\text {flip }} \approx 1.635$, as can be seen in Fig.5(c). Economically, the main reason for the occurrence of a periodtwo cycle is that the real side of the housing market, here in the form of the inverse cost parameter $\gamma$, leads to a very sharp reaction of the housing stock. Of course, a similar outcome may be observed if parameter $\beta$, capturing the sensitivity of the rental market, increases. Note that we assume in these simulations that homebuyers believe in mean reversion, i.e. $\phi<0$.

\section{Conclusions}

In this paper, we have reconsidered the conditions which guarantee that the three roots of a third-degree polynomial are inside the unit circle of the complex plane. We have shown that only three conditions are related to the boundary of the stability region, and that each condition is also related to a particular transition 
of the roots, exiting from the unit circle. These conditions and findings are particularly important in the applied context, when the dynamics of a three-dimensional map are of interest. In fact, these conditions are related to specific bifurcations. Each point of the boundary is associated with a bifurcation of codimension-1, -2 or -3 , as shown in the theorems described in Sec. 1, and proved in Sec. 2. When condition $(i)$ is violated, an eigenvalue equal to +1 is crossed, which represents a fold bifurcation, a transcritical bifurcation or a pitchfork bifurcation. When condition ( $i i)$ is violated, an eigenvalue equal to -1 is crossed, which represents a flip bifurcation. When condition (iii) is violated, a pair of complex conjugate eigenvalues becomes larger than 1 in modulus, which may represent a Neimark-Sacker bifurcation, and is thus associated with cyclic behaviors of the dynamics. In all the cases, all the three eigenvalues are explicitly given.

As an application to economics, in Sec. 3 we have considered a simple housing market model, illustrating the dynamics occurring when the three above-mentioned transitions occur. In particular, when condition $(i)$ is violated, the considered system undergoes a pitchfork bifurcation; when condition $(i i)$ is violated, the system undergoes a supercritical flip bifurcation, leading to an attracting cycle of period 2; while when condition (iii) is violated, a supercritical Neimark-Sacker bifurcation occurs, leading to attracting closed invariant curves. The latter scenario is particularly relevant for explaining the dynamics of housing markets, and our theorems allow us to prove rigorously under which economic conditions such an outcome may appear.

\section{Appendix}

\section{Proof of Lemma 1 (roots of a cubic polynomial).}

Since we know one real root $\xi_{1}$ of the polynomial, we can factorize $P(x)$ to determine the quadratic equation leading to the other pair of roots. From the equality

$$
P(x)=x^{3}+a_{1} x^{2}+a_{2} x+a_{3}=\left(x-\xi_{1}\right)\left(x^{2}-\tau x+\delta\right)
$$

knowing that $\xi_{1}^{3}+a_{1} \xi_{1}^{2}+a_{2} \xi_{1}+a_{3}=0$, we get:

$$
\tau=-\left(a_{1}+\xi_{1}\right)\left(=\xi_{2}+\xi_{3}\right), \delta=a_{2}+\xi_{1}\left(a_{1}+\xi_{1}\right)\left(=\xi_{2} \xi_{3}\right), a_{3}=-\xi_{1} \delta
$$

that is

$$
\tau=-\left(a_{1}+\xi_{1}\right), \delta=-\frac{a_{3}}{\xi_{1}}
$$

Hence, the other two roots are given by

$$
\xi_{2,3}=\frac{1}{2}(\tau \pm \sqrt{\Delta})
$$

where

$$
\Delta=\tau^{2}-4 \delta=a_{1}^{2}-2 a_{1} \xi_{1}-\left(3 \xi_{1}^{2}+4 a_{2}\right)
$$

These two roots are complex conjugate when $\Delta<0$, real and distinct when $\Delta>0$, and real and coincident when $\Delta=0$.

Considering that $\Delta=0$ occurs for

$$
a_{1, \pm}=\xi_{1} \pm 2 \sqrt{\xi_{1}^{2}+a_{2}}
$$

we have that

-if $\xi_{1}^{2}+a_{2}<0$, then $\Delta>0$ and two distinct real roots exist, given by $\xi_{2,3}=\frac{1}{2}(\tau \pm \sqrt{\Delta})$;

-if $\xi_{1}^{2}+a_{2}=0$, then $\Delta=\left(a_{1}-\xi_{1}\right)^{2}$ and two distinct real roots exist, given by $\xi_{2,3}=\frac{1}{2}\left(\tau \pm\left|a_{1}-\xi_{1}\right|\right)$, so that $\xi_{2}=-\xi_{1}$ and $\xi_{3}=-a_{1}$;

-if $\xi_{1}^{2}+a_{2}>0$ and

- $\left(\xi_{1}-2 \sqrt{\xi_{1}^{2}+a_{2}}\right)<a_{1}<\left(\xi_{1}+2 \sqrt{\xi_{1}^{2}+a_{2}}\right)$, then $\Delta<0$ and two complex conjugate roots exist, given by $\xi_{2,3}=\alpha \pm i \beta$, where $\alpha=\frac{\tau}{2}, \beta=\frac{\sqrt{4 \delta-\tau^{2}}}{2}=\sqrt{\delta-\alpha^{2}}$,

- $a_{1}=\left(\xi_{1}-2 \sqrt{\xi_{1}^{2}+a_{2}}\right)$ or $a_{1}=\left(\xi_{1}+2 \sqrt{\xi_{1}^{2}+a_{2}}\right)$, then $\Delta=0$ and two coincident real roots exist, given by $\xi_{2}=\xi_{3}=\frac{\tau}{2}=-\frac{a_{1}+\xi_{1}}{2}$, 
- $a_{1}<\left(\xi_{1}-2 \sqrt{\xi_{1}^{2}+a_{2}}\right)$ or $a_{1}>\left(\xi_{1}+2 \sqrt{\xi_{1}^{2}+a_{2}}\right)$, then $\Delta>0$ and two distinct real roots exist, given by $\xi_{2,3}=\frac{1}{2}(\tau \pm \sqrt{\Delta})$

Acknowledgements: Gardini, Sushko and Tramontana conducted this study within the research project on Models of Behavioral Economics for Sustainable Development, at the Department of Economics, Society, Politics (DESP) of the University of Urbino.

\section{References}

Agliari, A., Gardini, L. \& Puu, T. (2000). The dynamics of a triopoly Cournot game. Chaos, Solitons \& Fractals, 11, 2531-2560.

Agliari, A., Bischi, G. I., Dieci, R. \& Gardini, L. (2005). Global bifurcations of closed invariant curves: A computer assisted study. International Journal of Bifurcation and Chaos, 15(4) 1285-1328.

Anufriev, M. \& Bottazzi, G. (2010). Market equilibria under procedural rationality. Journal of Mathematical Economics, 46, 1140-1172.

Asano, T. \& Yokoo, M. (2019). Chaotic dynamics of a piecewise linear model of credit cycles. Journal of Mathematical Economics, 80, 9-21.

Bischi, G. I., Chiarella, C., Kopel, M. \& Szidarovsky, F. (2009). Nonlinear Oligopolies: Stability and Bifurcations, Springer-Verlag, New York.

Bischi, G. I. \& Tramontana, F. (2010). Three-dimensional discrete-time Lotka-Volterra models with an application to industrial clusters. Communications in Nonlinear Science and Numerical Simulation, 15(10), 3000-3014.

Brock, W. \& Hommes, C. (1997). A rational route to randomness. Econometrica, 65, 1059-1095.

Brock, W., Hommes, C. \& Wagener, F. (2005). Evolutionary dynamics in markets with many trader types. Journal of Mathematical Economics, 41, 7-42.

Case K. \& Shiller R. (2003). Is there a bubble in the housing market?. Brookings Papers on Economic Activity, 2, 299-362.

Case, K., Shiller, R. \& Thompson, A. (2012). What have they been thinking? Homebuyer behavior in hot and cold markets. Brookings Papers on Economic Activity, 2, 265-298.

Day, R. (1994). Complex economic dynamics: an introduction to dynamical systems and market mechanisms. MIT Press, Massachuchetts.

Cavalli, F., Naimzada, A. \& Pireddu, M. (2017). An evolutive financial market model with animal spirits: imitation and endogenous beliefs. Journal of Evolutionary Economics, 27, 1007-1040.

Dieci, R. \& Westerhoff, F. (2010). Heterogeneous speculators, endogenous fluctuations and interacting markets: a model of stock prices and exchange rates. Journal of Economic Dynamics and Control, 34, 743-764.

Dieci, R. \& He, X.-Z. (2018). Heterogeneous agent models in finance. In: Hommes, C. and LeBaron, B. (Eds.): Handbook of Computational Economics, 4, Heterogeneous Agent Modeling. North-Holland, Amsterdam, 257-328.

Dieci, R. \& Westerhoff, F. (2016). Heterogeneous expectations, boom-bust housing cycles, and supply conditions: a nonlinear economic dynamics approach. Journal of Economic Dynamics and Control, 71, 21-44.

Farebrother, R. W. (1973). Simplified Samuelson conditions for cubic and quartic equations. The Manchester School of Economics and Social Studies, 41(4), 396-400.

Farebrother, R. W. (2012). Sufficient conditions for the stability of linear difference equations. The Manchester School, 80(5) 630-632.

Galor, O. (2007). Discrete dynamical systems. Springer, Berlin.

Gandolfo, G. (2009). Economic Dynamics. Heidelberg: Springer.

Gaunersdorfer, A., Hommes, C. \& Wagener, F. (2008). Bifurcation routes to volatility clustering under evolutionary learning. Journal of Economic Behavior \&3 Organization, 67(1), 27-47

Guckenheimer, J. \& Holmes, P. (2002). Nonlinear oscillations, dynamical systems and bifurcations of vector fields, Springer, New York. 
Hens, T. \& Schenk-Hoppé, K. R. (2009). Handbook of financial markets: dynamics and evolution. NorthHolland, Amsterdam.

Hommes, C. (2013). Behavioral rationality and heterogeneous expectations in complex economic systems. Cambridge University Press, Cambridge.

Hommes, C. \& LeBaron, B. (2018). Handbook of computational economics: heterogeneous agent modeling. North-Holland, Amsterdam.

Invernizzi, S. \& Medio, A. (1991). On lags and chaos in economic dynamic models. Journal of Mathematical Economics, 20, 521-550.

Kuznetsov, Yu. A. (2004). Elements of applied bifurcation theory, Springer, Berlin.

Lines, M. (2005). Intertemporal equilibrium dynamics with a pollution externality. Journal of Economic Behavior and Organization, 56, 349-364.

Lines, M. \& Westerhoff, F. (2010). Inflation expectations and macroeconomic dynamics: the case of rational versus extrapolative expectations. Journal of Economic Dynamics and Control, 34, 246-257.

Lines M., Schmitt, N. \& Westerhoff, A. (2019). Stability conditions for three-dimensional maps and their associated bifurcation types. Applied Economics Letters (in press).

Miao, J. (2014). Economic dynamics in discrete time. MIT Press, Cambridge.

Matsumoto, A. \& Szidarovszky, F. (2014). Discrete-time delay dynamics of boundedly rational monopoly. Decisions in Economics and Finance, 37, 53-79.

Matsuyama, K. (1999). Growing through cycles. Econometrica, 67, 335-347.

Matsuyama, K., Sushko, I. \& Gardini, L. (2016). Revisiting the model of credit cycles with good and bad projects. Journal of Economic Theory, 163, 525-556.

Naimzada, A. \& Tramontana, F. (2015). Two different routes to complex dynamics in an heterogeneous triopoly game. Journal of Difference Equations and Applications, 21(7), 1-11.

Okuguchi, K. \& Irie, K. (1990). The Schur and Samuelson conditions for a cubic equation. The Manchester School, 58, 414-418.

Poterba, J. (1984). Tax subsidies to owner-occupied housing: An asset market approach. Quarterly Journal of Economics, 99, 729-752.

Poterba, J. (1991). House price dynamics: the role of tax policy and demography. Brookings Papers on Economic Activity, 2, 143-203.

Puu, T. (2004). Attractors, Bifurcations \& Chaos: Nonlinear Phenomena in Economics. Springer, Berlin.

Puu, T. \& Sushko, I. (2006). Business cycle dynamics: models and tools. Springer, Berlin.

Rosser, J. B. Jr. (2009). Handbook of research on complexity. Edward Elgar, Cheltenham.

Schmitt, N. \& Westerhoff, F. (2019). Short-run momentum, long-run mean reversion and excess volatility: an elementary housing model. Economics Letters, 176, 43-46.

Schmitt, N. \& Westerhoff, F. (2020). Speculative housing markets and rent control: insights from nonlinear economic dynamics. Working Paper, University of Bamberg.

Shone, R. (2002). Economic dynamics. Cambridge University Press, Cambridge.

Sorger, G. (2015). Dynamic economic analysis. Cambridge University Press, Cambridge.

Tesfatsion, L. \& Judd, K. (2006). Handbook of computational economics: agent-based computational economics. North-Holland, Amsterdam.

Tramontana, F., Gardini, L., Dieci, R. \& Westerhoff, F. (2010). Global bifurcations in a three-dimensional financial model of bull and bear interactions. In: Bischi, G.I., Chiarella, C. and Gardini, L. (Eds): Nonlinear Dynamics in Economics, Finance and Social Sciences. Springer, Berlin, Heidelberg, 333-352.

Tuinstra, J., Wegener, M. \& Westerhoff, F. (2014). Positive welfare effects of trade barriers in a dynamic partial equilibrium model. Journal of Economic Dynamics and Control 48, 246-264. 